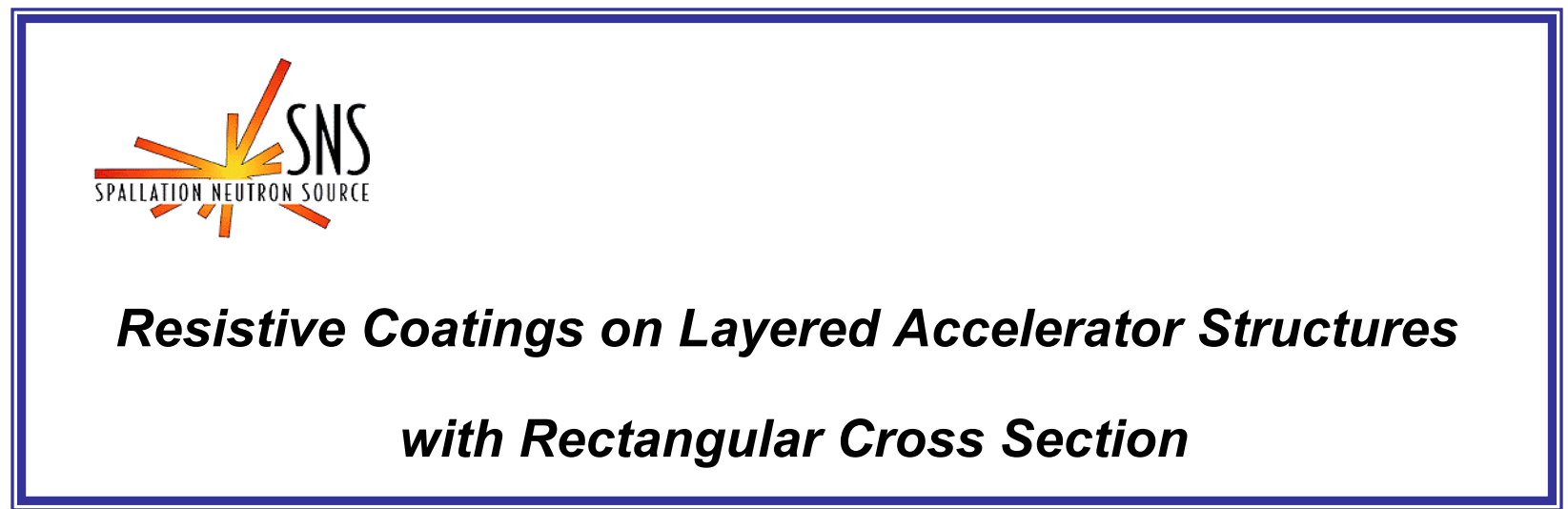

BNL/SNS TECHNICAL NOTE

NO. 103

H. Hahn and D. Davino

January 8, 2002

COLLIDER-ACCELERATOR DEPARTMENT BROOKHAVEN NATIONAL LABORATORY

UPTON, NEW YORK 11973 


\title{
Resistive Coatings on Layered Accelerator Structures with Rectangular Cross Section
}

\author{
H. Hahn and D. Davino \\ Brookhaven National Laboratory, Upton, NY 11973
}

\begin{abstract}
The coupling impedance of layered accelerator structures, typified by kicker magnets with rectangular cross section, is analyzed. Special attention is paid to the effects of a thin resistive coating layer as well as that of an external metallic bypass layer. It is shown that the lowest real part of the impedance is achieved with the combination of coating and bypass and that the bypass layer is required in reducing the impedance at very low frequencies. Furthermore, a comparison of estimates based on longitudinally uniform and finite length kicker structures indicates that the uniform structure yields acceptable estimates.
\end{abstract}

\section{Introduction}

The topic of resistive coating comes up in the design of any new accelerator or accelerator component. The typical structure consists of a ceramic beam tube inserted into a ferritic kicker magnet. Resistive coatings on the beam tubes or ferrite blocks of the kickers are applied for various reasons. Preventing electrostatic charging and reducing the secondary electron emission can be achieved with a high-resistance coating which does not affect the performance otherwise; in particular the rise time and the coupling impedance of a kicker remains unchanged. In contrast, the goal of avoiding wall heating by bunched beams or of minimizing the coupling impedance to avoid collective effects requires low-impedance coatings which can significantly impact the performance of a component and requires careful analysis.

The coupling impedance of layered/laminated structures and the effect of a resistive coating on the coupling impedance has, of course, been treated competently in several previous papers, notably by Zotter [1] and Piwinski [2], and in books [3,4]. In Piwinski's Handbook article [5], an expression for the coupling impedance is given for a ceramic beam tube with coating. This result indicates that a critical coating thickness equal to the square of the skin depth divided by the thickness of the ceramic wall is required in order to prevent field penetration and thus to reduce the coupling impedance. A fairly comprehensive theoretical and experimental study of the coupling impedance with high and low resistance beam tube coatings was done for the RHIC injection kicker [6]. The measured reduction in coupling impedance was in agreement with the theoretical predictions, although the final choice of high resistance was dictated by voltage holding requirements which is not addressed by theory. The rf screening by thin resistive layers was also confirmed by Caspers et.al [7]in coaxial wire measurements for the LHC dump 
kicker prototype. In a recent paper, Gluckstern and Zotter [8] presented an explanation based on wave reflections for the feature that a conducting layer thin compared to the skin depth is able to shield electromagnetic fields.

However, in spite of a vast array of papers, there remain several questions which require further work. One topic still being debated is the relative current distribution between resistive coating and metallic bypass and its effect on the transverse coupling impedance at very-low frequencies. Most results for layered structures assume longitudinal uniformity, and contain only a warning as to necessary modifications due to finite length or end effects. Standard scaling of the transverse from the longitudinal impedance is based on structures with circular cross section even though the typical kicker magnet is rectangular. Topics such as these are of current interest to the work on the SNS accumulator [9] and provided the stimulus for the present paper.

The approach here taken generally follows the previous publications, but differs in the detailed treatment and relies more on the results of an internal report by Hahn and Ratti [6], where the radial field penetration in a structure with concentric circular elements, representing rings with dielectric, ferrite, or metallic properties, is treated by a rigorous matrix method. However, since the coating thickness is much smaller than the radius of the ceramic beam tube, the local fields in the coating can be treated in a Cartesian coordinate system thereby avoiding Bessel functions with complex arguments. To keep the mathematical formalism simple and to focus on the physics of the problem, the treatment is here carried out entirely in a rectangular coordinate system which, in fact, is better suited for the geometry of kicker magnets. Further simplifications are achieved by considering the coupling impedance of a beam between infinite slabs with layers of different resistivity, permittivity or permeability, called the "plate" model $[10,11]$.

As first example, the exact expression for the resistive wall impedance in the plate model is derived in Section II, and its frequency dependence is numerically evaluated at typical energies. In Section III, the effect of a low-resistive coating layer on the impedance of a layered structure is discussed. For thin coatings the result can be stated, in qualitative terms, that the combined coupling impedance represents the structure and DC coating resistances in parallel. A practical expression for a thick metal layer, used as bypass, which remains valid a very low frequencies is then derived. In Section IV, the impedance of a multi-layered structure, representative of a ferrite kicker with external bypass and internal coating is presented. The possibility of a significant reduction of the resistive impedance part by a thin internal coating is demonstrated. Furthermore, in agreement with Caspers et al. experimental results, the bypass can carry current and further reduces the impedance at very low frequencies. In Section V, the changes introduced by the finite length of a structure are discussed and numerically compared to the infinite length solution. In the final Section VI, the necessary modifications of the plate model solution to accommodate a rectangular cross section are introduced and expressions for the longitudinal and transverse coupling impedance of layered, rectangular structures are given. 


\section{The Plate Model}

The coupling impedance of an accelerator structure is fully described by its surface (or Rsquare) impedance at the surface exposed to the beam. The case of the resistive wall impedance in a circular beam tube of radius $r_{B}$ and length $l_{B}$ with uniform surface properties represents the prototypical example [12],

$$
Z_{\|} \approx \frac{1}{2 \pi r_{B}} \frac{\mu \omega \delta}{2}=\frac{\mathbb{R}}{2 \pi r_{B}}
$$

with $Z_{\|}$the longitudinal coupling impedance per unit length and the frequency dependent skin depth $\delta$ and complex R-square value,

$$
\delta=\sqrt{\frac{2}{\mu \omega \sigma}} ; \mathbb{R}=(1+j) \sqrt{\mu \omega / 2 \sigma}
$$

This result is valid for metal tubes with walls thicker than the skin depth. The transverse coupling impedance is now derived by standard scaling as [13]

$$
Z_{\perp}=\frac{2 c}{\omega r_{B}^{2}} Z_{\|} .
$$

However, the analysis of the impedance of multi-layer walls, in particular the effect of a thin coating at low frequencies, requires a rigorous treatment, the details of which are given in the next section of this report. An understanding of the physics involved can best be gained by developing the heuristic solution for a simple model of a current sheet between two plates ("slabs") as shown in Fig.1. This model can locally represent a generic device, such as the resistive wall beam tube or a kicker magnet, by retaining the value for the surface impedance and equating the spacing of the current sheet to wall, $b$, with the radius $r_{B}$. The coating of thickness $d$ is now added to the plate but without changing the plate's R-square impedance value. The coupling impedance seen by the beam is still determined by the R-square impedance of the plate but screened by the coating. Analyzing the effect of a resistive coating on the surface resistance can therefore be done in the plate model and the result is then applied directly to the beam tube impedance, provided that the coating thickness is small compared to the beam tube radius.

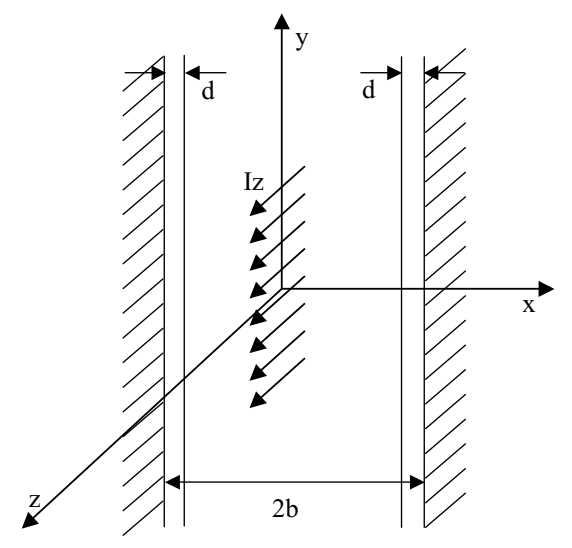

Fig.1. Geometry for the skin effect analysis 
The analysis of the plate model starts with the electromagnetic fields being generated by a current sheet

$$
i_{z}(0)=\mathfrak{I} \delta(0) e^{-j k z+j \omega t}
$$

with $\mathfrak{I}=I / h$ representing the current $I$ extended vertically over $h$.

The fields in each of the structure layers are transverse magnetic and can be obtained from a scalar potential, solution of

$$
\nabla^{2} u+k^{\prime 2} u=0
$$

according to

$$
\begin{aligned}
& E_{z}=\frac{\partial^{2} u}{\partial z^{2}}+k^{\prime 2} u \\
& H_{y}=-j \omega \varepsilon^{\prime} \frac{\partial u}{\partial x}
\end{aligned}
$$

with $k^{\prime 2}=\mu \varepsilon^{\prime}(\omega / c)^{2}$ and $\varepsilon^{\prime}=\varepsilon-j \sigma / \omega$.

The solutions are appropriate combinations of $e^{j \kappa^{\prime} x}, e^{-j \kappa^{\prime} x}$ with $\kappa^{\prime 2}=k^{2}-\mu \varepsilon^{\prime}(\omega / c)^{2}$.

The solution for the fields in the free space between current sheet and structure is found to be

$$
\begin{aligned}
& E_{z}(x)=j \frac{Z_{0} \mathfrak{I}}{2} \frac{c \kappa}{\omega} e^{-\kappa x}+\mathfrak{I} q_{i} \frac{\cosh \kappa x}{\cosh \kappa b} \\
& H_{y}(x)=\frac{\mathfrak{J}}{2} e^{-\kappa x}-j \mathfrak{I} \frac{q_{i}}{Z_{0}} \frac{\omega}{c \kappa} \frac{\sinh \kappa x}{\sinh \kappa b}
\end{aligned}
$$

with the common factor $e^{-j k z} e^{j \omega t}$ suppressed and

$$
\kappa^{2}=k^{2}-(\omega / c)^{2}=\left(\frac{\omega}{\beta \gamma c}\right)^{2}
$$

where $\beta$ and $\gamma$ are the usual kinematic variables. One finds $q_{i}$ from the ratio at the boundary of the beam aperture, $E_{z}(b) / H_{y}(b)=-\Re$ as

$$
q_{i}=-\frac{1}{2} \frac{\Re+j Z_{0} \frac{\kappa c}{\omega}}{1-j \frac{\omega \tanh \kappa b}{\kappa c} \frac{\Re}{Z_{0}}} e^{-\kappa b}
$$

Ignoring the space charge contribution, one finds the structure contribution to the coupling impedance per unit length,

$$
Z_{K}=-E_{z}(0) / I=-q_{i} /(h \cosh \kappa b) .
$$

In the limit of ultra-relativistic beams, $\kappa \rightarrow 0$, and thick metallic walls, $\Re=\mathbb{R} \ll Z_{0}$, one has

$$
Z \approx \mathbb{R} / 2 h
$$

The dependence of the coupling impedance on energy is shown in Fig. 2. It is seen, that the imaginary part is energy dependent, whereas above $\sim 1 \mathrm{GeV}$ the energy dependence of the real part is negligible and can be found from the skin effect resistance directly. 

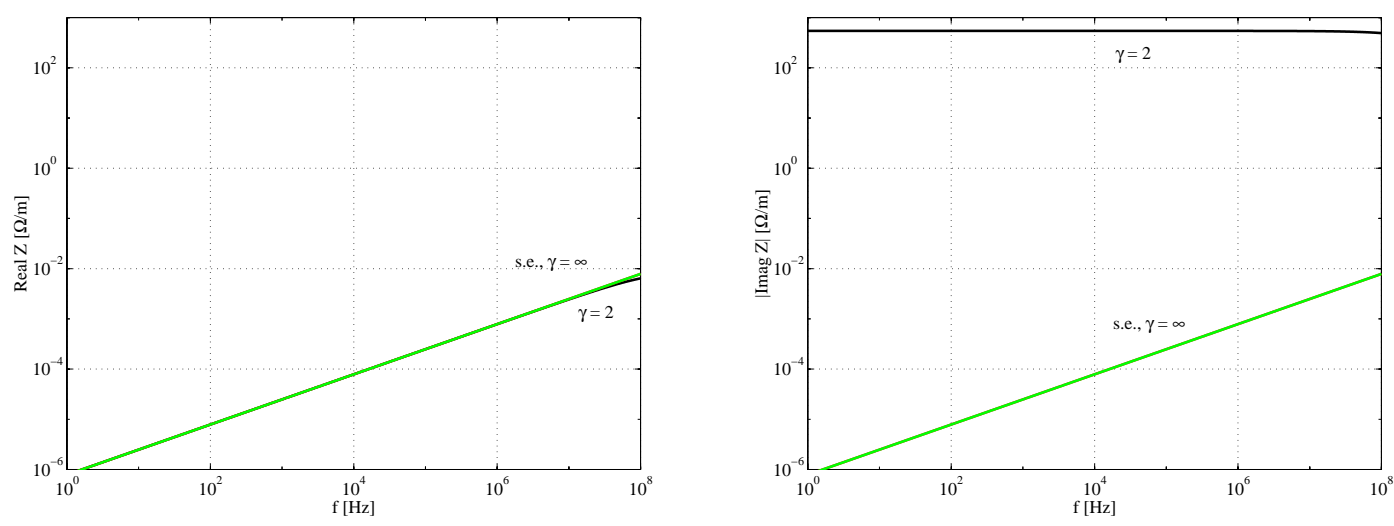

Fig.2. Energy dependence of the coupling impedance due to a resistive wall with s.e. representing the skin effect for $\sigma=40 \times 10^{6} / \Omega \mathrm{m}$

\section{Coating Layer}

The screening effect resulting from the coating layer is analyzed by considering the fields in the coating,

$$
\begin{aligned}
& E_{z i}=E_{z o} \cosh \kappa^{\prime} d-H_{y o} \mathbb{Z}^{\prime} \sinh \kappa^{\prime} d \\
& H_{y i}=-E_{z o} \mathbb{Z}^{\prime-1} \sinh \kappa^{\prime} d+H_{y o} \cosh \kappa^{\prime} d
\end{aligned}
$$

These equations relate the field pair at the inner to that at the outer side, identified by the subscript $i$ and $o$. For a typical conductive material one has $\omega \varepsilon \ll \sigma, \mu \approx \mu_{0}$, and $\varepsilon \approx \varepsilon_{0}$. In the high conductivity approximation one has $\varepsilon^{\prime} \approx-j \sigma / \omega$ leading to

$$
\kappa^{\prime} \approx \kappa_{\sigma}=\sqrt{j \mu \sigma \omega}=\frac{1+j}{\delta},
$$

and the conventional expressions for the skin depth, $\delta=\sqrt{\frac{2}{\mu \omega \sigma}}$,

and the "wave" impedance, $\mathbb{Z}^{\prime} \approx \mathbb{R}=\frac{\kappa_{\sigma}}{\sigma}=\frac{1+j}{2} \mu \omega \delta=\frac{1+j}{\delta \sigma}$.

Finally, since $E_{z o}=-\mathfrak{R}_{K} H_{y o}$ remains unchanged, the "screened" surface impedance seen by the beam follows rigorously as

$$
\Re=-\frac{E_{i}}{H_{i}}=\frac{\Re_{K}+\mathbb{R} \tanh \kappa_{\sigma} d}{1+\Re_{K} \mathbb{R}^{-1} \tanh \kappa_{\sigma} d}
$$

The coating layer can be characterized as "thin", if $d \ll \delta$. For a generic coating, with a conductivity of $\sigma=4 \times 10^{7} / \Omega \mathrm{m}$ and $10 \mu \mathrm{m}$ thick, this limit implies frequencies below $\sim 60 \mathrm{MHz}$. At "low" frequencies, i.e. for $d / \delta<1$, the term $\mathbb{R} \tanh \kappa_{\sigma} d$ approaches the inductance of the coating, $j \mu \omega d$ and thus is negligible with respect to typical structure impedances. The other term $\mathbb{R}^{-1} \tanh \kappa_{\sigma} d \rightarrow \sigma d$ and represents the inverse dc 
$\mathrm{R}$-square impedance of the coating. It follows that the "screened" surface impedance is obtained in good approximation as the parallel assembly of $\Re_{K}^{-1}$ and $\sigma d[6]$,

$$
\Re \approx \frac{1}{\mathfrak{R}_{K}^{-1}+\sigma d}
$$

It is instructive to apply the general result to a "thick" metallic layer alone in free space without external layer, as typified by the bypass. The free space impedance for air differs from vacuum by the non-zero electric susceptibility, $\varepsilon_{r}-1 \approx 6 \times 10^{-4}$, and is given by

$$
\Re_{o}=-j Z_{0} \kappa / \omega=-j Z_{0} \sqrt{\frac{1}{(\beta \gamma)^{2}}-6 \times 10^{-4}} .
$$

Although the difference seems small, it can result in qualitatively different effects at energies above the critical value $\gamma_{c} \approx 40$, in particular it results in Cerenkov radiation. [14] The dependence on beam energy was numerically investigated with the results shown in Fig.3. On sees that in the frequency range of interest, the R-square value of a "thick" bypass is adequately represented by the standard skin effect impedance, $\mathbb{R}$.

Only at the lowest frequencies, the energy dependence becomes noticeable with energies below and above the critical $40 \mathrm{GeV}$ having essentially equal impedance values, given by the dc resistance of the layer. Only at the critical energy, where the external impedance is zero, and thereby reflecting the often used case of infinite conductance, the values are different. This suggests that the R-square impedance of a "thick" outer metal layer, here referred to as bypass, is over the entire frequency range adequately described by the simple expression, $\mathbb{R} / \tanh \kappa_{\sigma} d_{\sigma}$, which is energy independent.
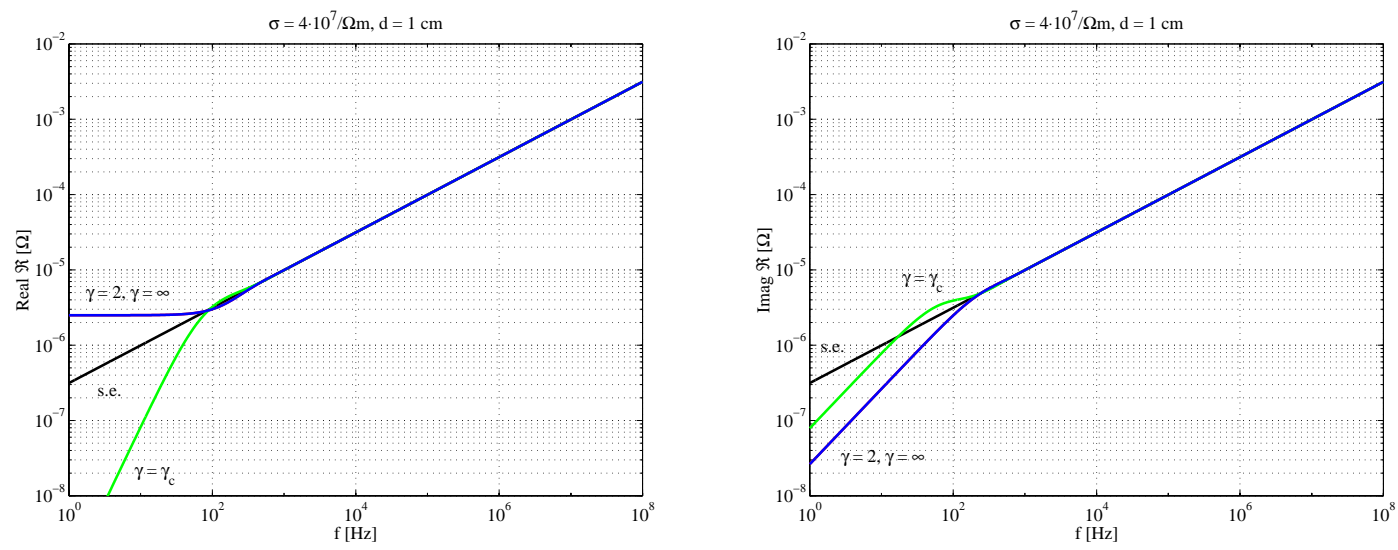

Fig. 3. R-square impedance of a metallic bypass with generic properties, comparing the results for finite thickness with the conventional skin effect values 


\section{Multi-Layer Structure}

The case of a general structure such as a ferrite layer with an external "bypass" metal and inner "screening" coating is of immediate interest and, in fact, provided the stimulus for the present report.

A matrix method is here presented to obtain theoretical expressions for the longitudinal coupling impedance of an accelerator component which can be represented by the simple model constructed with several layers of plates [6]. The coupling impedance is generated by the beam-excited TM mode electromagnetic fields, propagating in the center of the structure in the longitudinal direction. The calculation of the longitudinal coupling impedance seen by a beam can be reduced to the determination of an equivalent surface (R-square) impedance at the innermost side of the structure. In view of the above results showing only a weak energy dependence of the coupling impedance, it is often sufficient to address only the ultra-relativistic case. However, the treatment of the free space outside the structure entails conceptual problems and thus its energy dependence is considered in this Section.

The transverse field propagation in a laminated plate structure with arbitrary properties, including resistive, dielectric and/or magnetic, is fully described by the knowledge of the longitudinal electric and vertical magnetic field at the inner and outer plane of each layer. The concept of surface impedance now greatly facilitates the analysis of propagation through a series of layers, since it is basically defined as the ratio of electric and magnetic field strength at a given plane and thus provides the formal analogy between wave propagation and conventional low frequency transmission line theory.

The expressions for the fields of interest within a layer of uniform properties can be derived from a scalar potential based on the hyperbolic functions as described in the preceding section. The above equations lend themselves to a matrix notation which simplifies the analysis of a structure with regions of different properties. The fields at the inner and outer plane of a layer are related by the matrix

$$
\left(\begin{array}{l}
E_{z}\left(x_{i}\right) \\
H_{y}\left(x_{i}\right)
\end{array}\right)=\left[\begin{array}{ll}
M_{e e} & M_{e h} \\
M_{h e} & M_{h h}
\end{array}\right]\left(\begin{array}{l}
E_{z}\left(x_{o}\right) \\
H_{y}\left(x_{o}\right)
\end{array}\right)
$$

with the expressions for the matrix elements (in natural units, $c=\mu_{0}=1, Z_{0}=1$ )

$$
\begin{aligned}
& M_{e e}=\cosh \left(\kappa^{\prime} d\right) \\
& M_{e h}=-\mathbb{Z}^{\prime} \sinh \left(\kappa^{\prime} d\right) \\
& M_{h e}=-\mathbb{Z}^{\prime-1} \sinh \left(\kappa^{\prime} d\right) \\
& M_{h h}=\cosh \left(\kappa^{\prime} d\right)
\end{aligned}
$$

with the common factor $e^{-j k z} e^{j \omega t}$ suppressed and $d=x_{o}-x_{i}$. The other symbols represent the characteristic impedance of the layer, 


$$
\mathbb{Z}^{\prime}=\frac{\kappa^{\prime}}{j \varepsilon^{\prime} \omega}
$$

and the horizontal wave number

$$
\kappa^{\prime 2}=k^{2}-\mu \varepsilon^{\prime} \omega^{2}=\left(\frac{1}{\beta^{2}}-\mu \varepsilon^{\prime}\right) \omega^{2}
$$

where $\varepsilon^{\prime}=\varepsilon-j \sigma / \omega$ with the normalized beam velocity $\beta$, and the relative dielectric and permeability constants, $\varepsilon$ and $\mu$, and the conductivity $\sigma$.

For convenience sake, the matrix for a metallic layer is here explicitly given,

$$
M_{\sigma}=\left[\begin{array}{cc}
\cosh \kappa_{\sigma} d_{\sigma} & -\mathbb{R} \sinh \kappa_{\sigma} d_{\sigma} \\
-\mathbb{R}^{-1} \sinh \kappa_{\sigma} d_{\sigma} & \cosh \kappa_{\sigma} d_{\sigma}
\end{array}\right]
$$

with the symbols $\mathbb{R}$ and $\kappa_{\sigma}$ as defined above. The matrix for a ferrite/dielectric material follows as

$$
M=\left[\begin{array}{cc}
\cos \kappa_{\mu \varepsilon} d & -j \mathbb{Z}_{\mu \varepsilon} \sin \kappa_{\mu \varepsilon} d \\
-j \mathbb{Z}_{\mu \varepsilon}^{-1} \sin \kappa_{\mu \varepsilon} d & \cos \kappa_{\mu \varepsilon} d
\end{array}\right]
$$

with the wave number and impedance of the material

$$
\kappa_{\mu \varepsilon}^{2}=\left(\mu \varepsilon-\frac{1}{\beta^{2}}\right) \omega^{2} \& \mathbb{Z}_{\mu \varepsilon}=\frac{1}{\varepsilon} \sqrt{\mu \varepsilon-\frac{1}{\beta^{2}}}
$$

Furthermore, the matrix for a layer in free space is

$$
M=\left[\begin{array}{cc}
\cosh (\omega d / \beta \gamma) & j \frac{1}{\beta \gamma} \sinh (\omega d / \beta \gamma) \\
-j \beta \gamma \sinh (\omega d / \beta \gamma) & \cosh (\omega d / \beta \gamma)
\end{array}\right]
$$

The total matrix representing the sequence of layers in a structure is now obtained by appropriate matrix multiplication. The matrix relates electric and magnetic fields rather than voltage and current, but continuity of radial power flow requires that its determinant is still unity, $\operatorname{det}(M)=1$.

Taking into account the surface impedance (or R-square) definition at distance $\mathrm{x}$,

$$
\Re(x)=-\left(E_{z}(x) / H_{\varphi}(x)\right)
$$

one obtains the surface impedance transformation from outer to inner plane as

$$
\mathfrak{R}\left(x_{i}\right)=\frac{\mathfrak{R}\left(x_{o}\right) M_{e e}-M_{e h}}{M_{h h}-\mathfrak{R}\left(x_{o}\right) M_{h e}} .
$$

In the usual case, when there is air outside the accelerator structure, one finds the surface impedance at the structure outside to be (natural units)

$$
\Re\left(x_{o}\right)=-j \frac{\kappa}{\omega} \approx-j \sqrt{\frac{1}{(\beta \gamma)^{2}}-6 \times 10^{-4}}
$$


The foregoing matrix treatment can be handled without special difficulty by way of computer programs, including complex arguments due to lossy materials, and it is informative to apply the full formalism to the two representative case of a loss-less dielectric layer and also to a lossy ferrite layer. The relative effects due to an internal metallic thin coating and/or a thick bypass are readily evaluated over a broad frequency range, including the low- frequency end down to $1 \mathrm{~Hz}$.

Ceramic Layer. The various configurations with respect to coating and bypass are numerically evaluated for a generic loss-less ceramic structure with $\varepsilon=10, d_{\varepsilon}=3 \mathrm{~cm}$ and the results for the real part and imaginary part of the inner surface impedance are shown in Fig. 4

The real part of the impedance is of particular importance to the beam stability. The analysis of the numerical results points to some interesting facts:

- The naked ceramic layer, without coating and without bypass, exhibits the Cerenkov effect at energies above the critical $40 \mathrm{GeV}$.

- The impedance of the structure with coating alone or bypass alone is at sufficiently high frequencies, here above $10 \mathrm{kHz}$, independent of energy

- At energies not close to the critical value, the impedance of a coated ceramic shows a resistive component equal to its $\mathrm{DC}$ value over the entire frequency range of interest

- Surprisingly, at energies away from the critical value, the lowest resistive impedance value is obtained with the bypass at the ceramic outside.

- The impedance of the ceramic shows at the critical energy a significantly different behavior at low frequencies, here below $10 \mathrm{kHz}$, suggesting that the use of a "perfect" short at the outer wall is at most energies, especially at ultra-relativistic energies, not justified.

Ferrite Layer. The corresponding configurations for a ferrite structure with $d_{\mu}=3 \mathrm{~cm}$ and a realistic permeability (CMD5005), $\mu=1+\frac{1830}{1+j f / 2.23 \mathrm{MHz}}, \varepsilon=10$, were evaluated and the results are shown in Fig. 5. Comments regarding the real impedance are in line with those for the dielectric layer. However, the presence of ferrite losses especially at higher frequencies is evident, and points to the necessity of the coating layer.

The possibility of reducing the impedance with a combination of coating and bypass exists, but is limited to extremely low frequencies, here $\sim 10 \mathrm{kHz}$. As expected, the Cerenkov impedance at high energies shows up in the naked structure as well. The impedance at the critical energy is again qualitatively different from that at low energies. With respect to the imaginary part of the impedance, it suffices to mention that the high mu of the ferrite leads to resonant behavior in the frequency range of interest.

General Comments. The customary use of a bypass with infinite conductivity or the treatment of the external space as perfect vacuum leads, in general, to qualitatively 
erroneous results at low frequencies, and is only justified in the vicinity of the critical energy at about $40 \mathrm{GeV}$. The presence of a bypass is adequately represented at all frequencies and over a broad range of energies by its R-square impedance, $\mathbb{R} / \tan \kappa_{\sigma} d_{\sigma}$, as shown above.
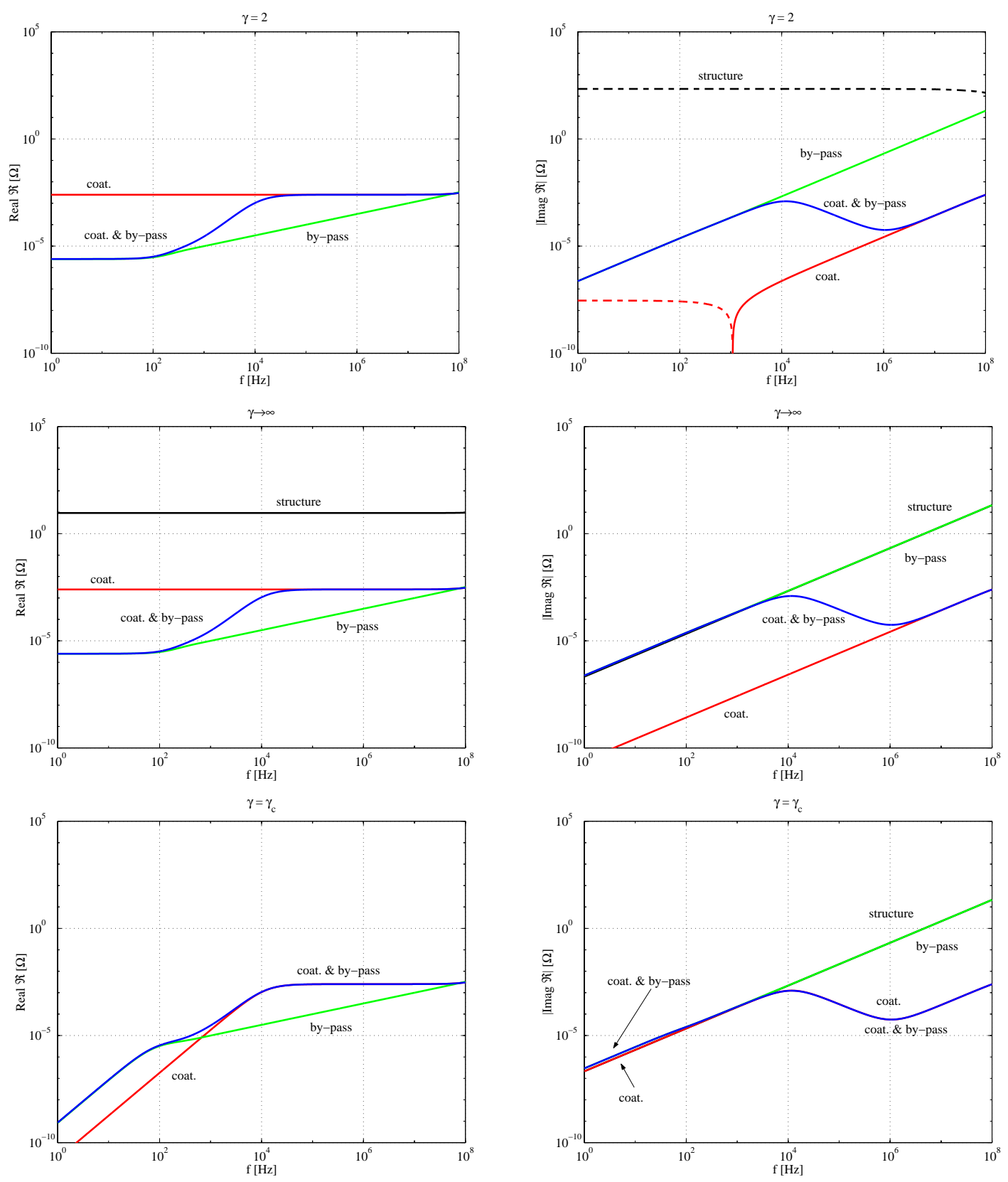

Fig.4. Inner surface impedance of a ceramic layer at various energies. 

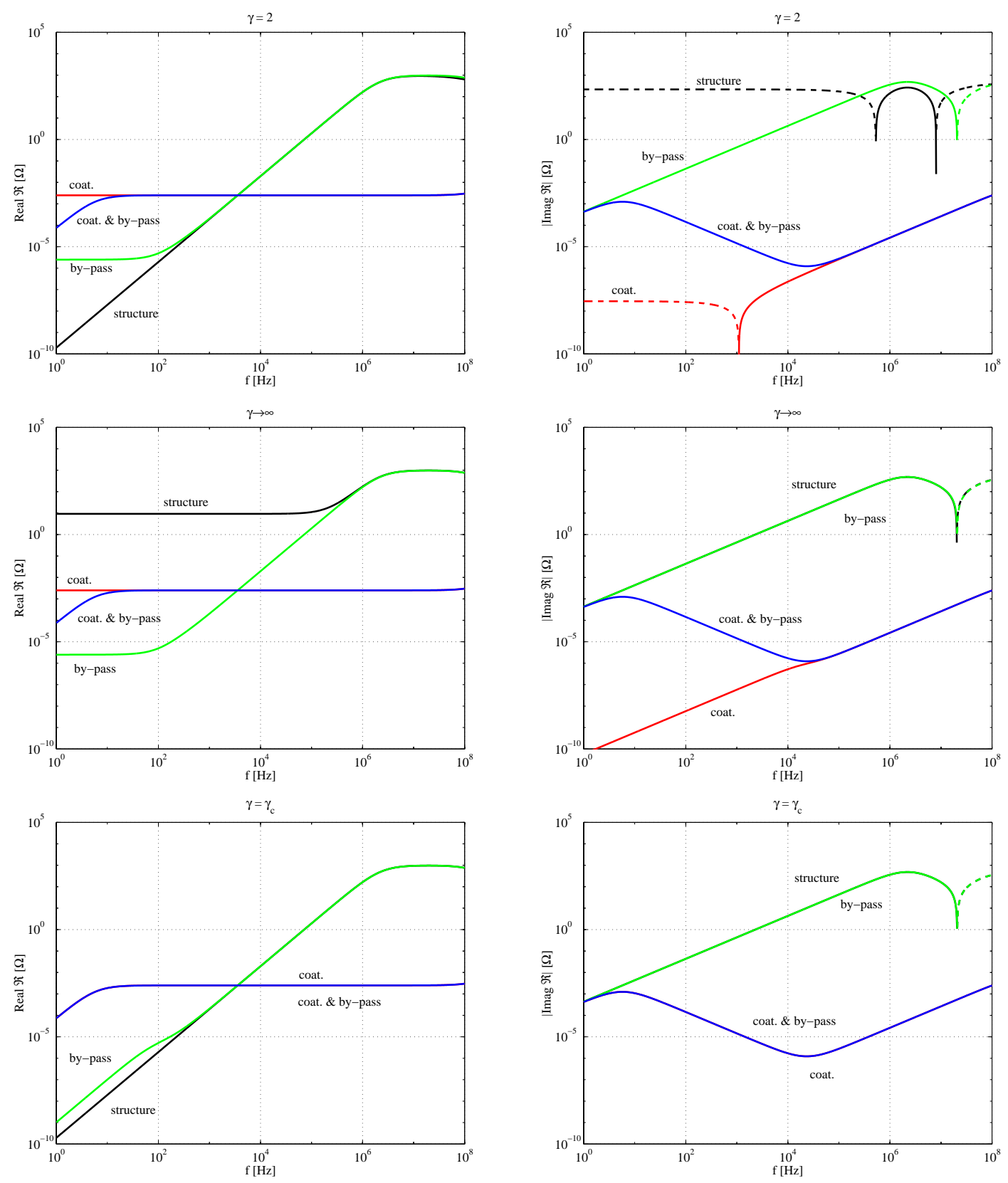

Fig. 5. Inner surface impedance of lossy ferrite at various energies. 


\section{Structures with finite length}

The R-square impedance of structure with finite length, $L$, can be derived by using the general expressions for the infinitely long case with appropriate modifications. It can be assumed that the inner coating and the bypass are perfectly connected to the loss-less beam tube. The field within the dielectric/ferrite layers of the structure is now expanded into an (infinite) set of stationary TM mode eigen-functions which involves replacing the $e^{j k z}$ dependence with

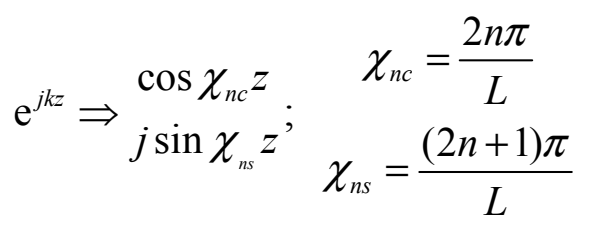

The matrices reflect the transverse wave propagation and thus retain essentially the same functional dependence but with changed $\kappa$-wave numbers in the dielectric/ferrite layers. Explicitly, the matrix associated with the $\mathrm{n}$-th mode in a dielectric/ferrite layer is

$$
\left[\begin{array}{cc}
\cos \kappa_{n} d_{\mu \varepsilon} & -j \mathbb{Z}_{n} \sin \kappa_{n} d_{\mu \varepsilon} \\
-j \mathbb{Z}_{n} \sin \kappa_{n} d_{\mu \varepsilon} & \cos \kappa_{n} d_{\mu \varepsilon}
\end{array}\right]
$$

with $\kappa_{n}=\omega \sqrt{\mu \varepsilon-\left(\chi_{n} / \omega\right)^{2}}$ and $\mathbb{Z}_{n}=\frac{1}{\varepsilon} \sqrt{\mu \varepsilon-\left(\chi_{n} / \omega\right)^{2}}$.

The transverse wave number is k-independent in the metallic layers and, therefore, remains unchanged. The Matrix of a layered structure follows by matrix multiplication as in the case of a uniform structure.

The effective surface impedance seen by the beam is now given by the sum of the impedances generated by each mode,

$$
\begin{aligned}
\Re= & \sum_{n} G_{n c}^{2} \frac{\Re_{o} M_{e e}\left(\kappa_{n c}\right)-M_{e h}\left(\kappa_{n c}\right)}{M_{h h}\left(\kappa_{n c}\right)-\Re_{o} M_{h e}\left(\kappa_{n c}\right)} \\
& +\sum_{n} G_{n s}^{2} \frac{\Re_{o} M_{e e}\left(\kappa_{n s}\right)-M_{e h}\left(\kappa_{n s}\right)}{M_{h h}\left(\kappa_{n s}\right)-\Re_{o} M_{h e}\left(\kappa_{n s}\right)}
\end{aligned}
$$

with the G-factors representing the coupling between the beam-induced coherent field and the stationary fields in the structure,

$$
\begin{aligned}
& G_{n c}=\int_{-L / 2}^{L / 2} \cos \chi_{n c} z e^{j k z} d z=\frac{(k L / 2) \sin (k L / 2)}{(n \pi)^{2}-(k L / 2)^{2}} \\
& G_{n s}=j \int_{-L / 2}^{L / 2} \sin \chi_{n s} z e^{j k z} d z \frac{(k L / 2) \cos (k L / 2)}{(n+1 / 2)^{2} \pi^{2}-(k L / 2)^{2}} .
\end{aligned}
$$

The R-square impedance at the outer plane of the structure is that of vacuum or air, and its correct value remains subject to discussion but since the fields are here stationary the wave impedance of free space $Z_{0}$ is an appropriate choice. In the presence of a bypass, the $\mathrm{R}$-square impedance is adequately approximated by

$$
\Re_{o}=\mathbb{R} / \tanh \kappa_{\sigma} d_{\sigma} .
$$


In order to test the convergence of the expression for the effective surface impedance, a $1 \mathrm{~m}$ long structure consisting of a $3 \mathrm{~cm}$ thick lossy ferrite with bypass was evaluated with an increasing number of terms. The results found at $100 \mathrm{MHz}$, where the need for many terms may be expected, is shown in Fig. 6 and suggest that convergence can be obtained with only a few terms. In the presence of a coating, the convergence will be even faster since the radial wave number in a resistive layer is independent of length.

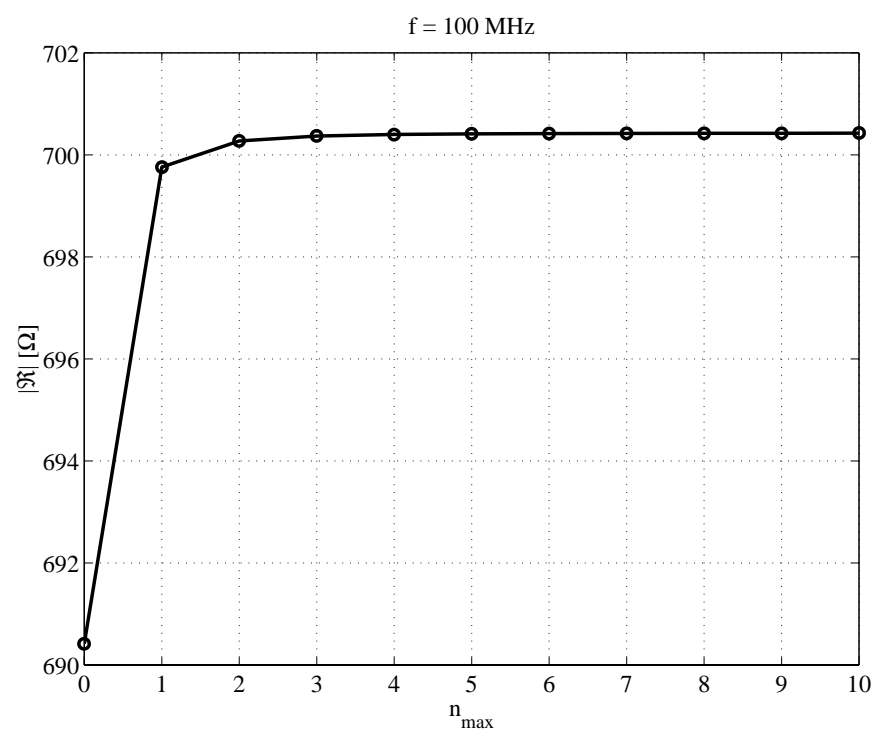

Fig.6. Convergence of the expression for the effective surface impedance

The difference between surface impedance values obtained for a uniform and finite length structure respectively was evaluated by means of $1 \mathrm{~m}$ long kicker model with $3 \mathrm{~cm}$ thick ferrite layer and a $1 \mathrm{~cm}$ bypass. The results for the two cases in Fig. 7 indicate that the use of the uniform approximation provides acceptable results up to the tens of $\mathrm{MHz}$ range.
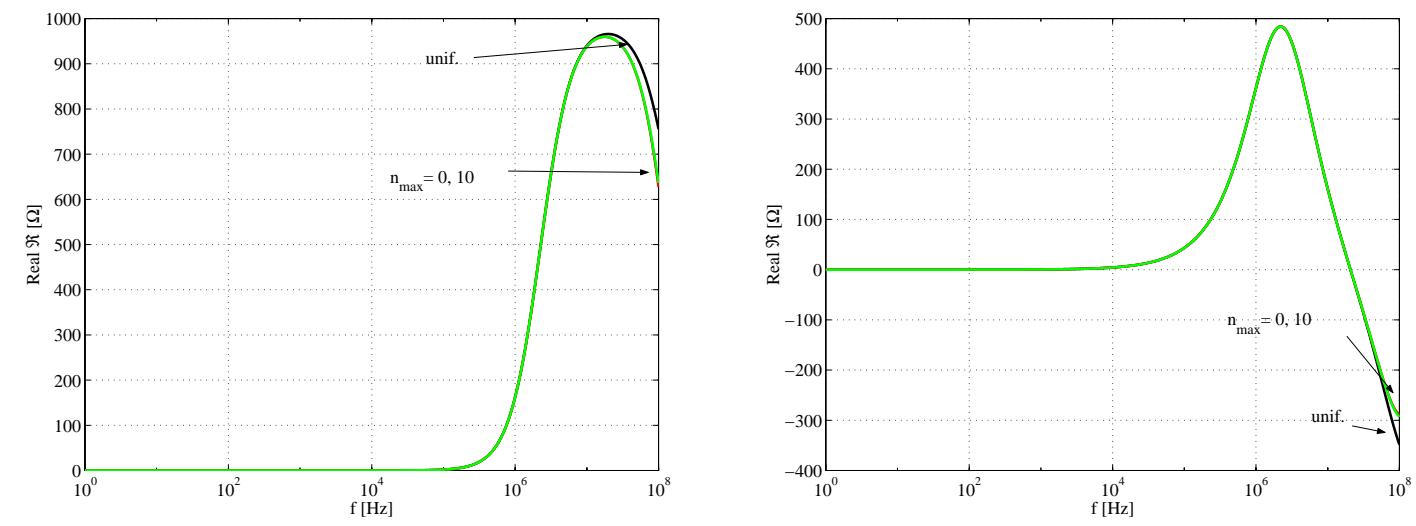

Fig. 7. The surface impedance of a kicker model with $3 \mathrm{~cm}$ ferrite and $1 \mathrm{~cm}$ bypass layers with the treatment as a uniform or a finite length structure. 
Changes of transverse dimensions in structures without ferrite or dielectric must be treated as single or double step discontinuity [15]. If the spacing between steps is larger than the step, each step presents at low frequencies an inductance. Obviously, the value of the step inductance cannot be found from the uniform solution of a wave guide. If the step is comparable to the length of the discontinuity, the interference between the step induced fields lead to pill-box resonances, which show up at higher frequencies and require finite length treatment $[7,8]$. Although the plate model is not intended for a pill box analysis, it still can be used to estimate its impedance in the presence of a coating layer.

\section{Coupling Impedance Estimates for Rectangular Cross Section at ultra relativistic energies}

In the preceding sections, the R-square or surface impedance of layered accelerator structures with infinite length was obtained in the plate model approximation and changes due to a finite length were analyzed. In this last section, estimates for the longitudinal and transverse coupling impedance of structures with rectangular cross section are derived by assuming that the vertical is (much) larger than the horizontal aperture. In this case, the fields generated by the beam are adequately defined by the plates with perfect conductivity. In the perturbative approach used here, the beam-induced magnetic field at the plate produces a longitudinal electric field and gives the estimate for the coupling impedance.

\section{Longitudinal coupling impedance}

The fields generated by an ultra-relativistic delta current of strength, $I$, between perfect plates is known from summation of mirrored currents [16] or directly from conformal mapping $(w=\tan z)[17]$ to be

$$
\begin{aligned}
& E_{x}(x, y)=H_{y}(x, y)=I \frac{\sin (\eta x) \cosh (\eta y)}{2 b(\cosh 2 \eta y-\cos 2 \eta x)} \\
& E_{y}(x, y)=-H_{x}(x, y)=I \frac{\cos \eta x \sinh \eta y}{2 b(\cosh 2 \eta y-\cos 2 \eta x)}
\end{aligned}
$$

where the common factor $e^{j \omega t-j k z}$ is omitted for convenience. The magnetic field at the plate produces a longitudinal electric field, which to first order is independent of the transverse position,

$$
E_{z}(b, y) \approx-\mathfrak{N} H_{y}(b, y) .
$$

The longitudinal electric field at the location of the beam is thus

$$
E_{z}(0,0) \approx-\frac{\Re}{4 b} e^{-j k z}
$$

In the limit of ultra-relativistic beams and the perturbation treatment used here, one finds the longitudinal coupling impedance per unit length of the structure with wall impedance $\mathfrak{R}$ as, 


$$
Z_{\|}=\frac{\Re}{4 b}
$$

The longitudinal coupling impedance of a double step discontinuity, simulating the pill box in ref. 7, $80 \mathrm{~cm}$ long with $50 \mathrm{~cm}$ i.d. and $5 \mathrm{~cm}$ beam tubes, is shown in Fig. 8 . The results are for thick metal walls alone and thick metal walls plus inner coating. The resonance although determined in the plate model corresponds to the $\mathrm{TM}_{010}$ mode of the pill box. Below this resonance, the open discontinuity (i.e. without coating) is determined by the skin effect resistance of the bypass wall and the inductance, i.e the $R /\left(Q \omega_{0}\right)$, of the resonance. Its coupling impedance can be represented by the two components in series. The coating serves as shunt to the discontinuity and suppresses the resonance. At very low frequencies, the impedance of the inductance tends to zero, and the remaining impedance is determined by the dc resistance of bypass and coating in parallel.
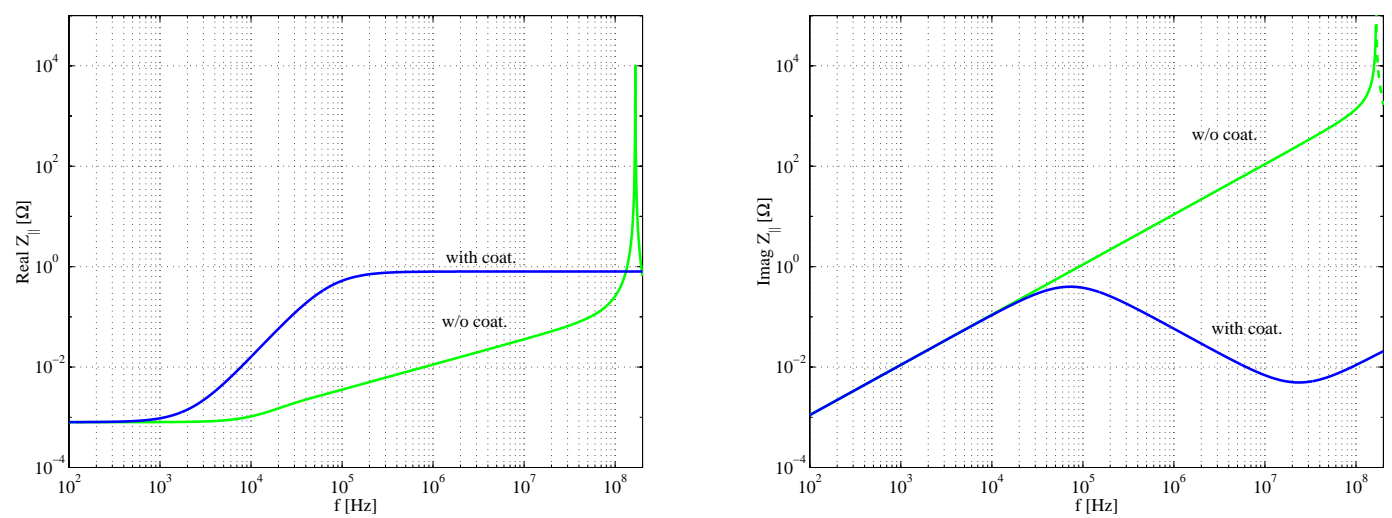

Fig. 8. Longitudinal coupling impedance of double step discontinuity

\section{Transverse coupling impedance}

The fields generated by a dipole current, $I \Delta$, between perfect plates is known from conformal mapping $(w=\log \tan z)$ to be

$$
\begin{aligned}
& E_{x}(x, y)=H_{y}(x, y)=I \Delta \frac{\pi}{16 b^{2}} \frac{1-\cos 2 \eta x \cosh 2 \eta y}{\left(\sin ^{2} \eta x+\sinh ^{2} \eta y\right)^{2}} \\
& E_{y}(x, y)=-H_{x}(x, y)=I \Delta \frac{\pi}{16 b^{2}} \frac{\sin 2 \eta x \sinh 2 \eta y}{\left(\sin ^{2} \eta x+\sinh ^{2} \eta y\right)^{2}}
\end{aligned}
$$

The resistive wall induces a longitudinal electric field on the median plane which is to first order given by

$$
E_{z}(x, 0) \approx-\Re \frac{x}{b} H_{y}(b, 0) e^{-j k z}
$$

Applying the Panofsky-Wenzel theorem leads to the transverse impedance per unit length, [18]

$$
Z_{\perp}=\Re \frac{\pi}{8 k b^{3}}
$$

Thus, the transverse coupling impedance in structures with rectangular cross section can be scaled from the longitudinal according to 


$$
Z_{\perp}=Z_{\|} \frac{\pi}{2 k b^{2}}
$$

in contrast to circular structures where one has

$$
Z_{\perp}=Z_{\|} \frac{2}{k b^{2}}
$$

The transverse coupling impedance of a rectangular structure, in the ultra relativistic limit, is shown in Fig. 9. The structure, $1 \mathrm{~m}$ long and $20 \mathrm{~cm}$ vertical aperture, consists of a $6 \mathrm{~mm}$ ceramic and $3 \mathrm{~cm}$ lossy ferrite layer. The impedance is obtained for the structure with the application of a $10 \mu \mathrm{m}$ resistive coating, a $1 \mathrm{~cm}$ bypass, or the combination of both .

The numerical results are qualitatively different from those of the double step discontinuity. Notable is the absence of a resonance, which is completely damped by ferrite losses, with the implication that the results could have been obtained from the uniform structure calculations. Potentially of concern is the $1 / \omega$ dependence of the transverse impedance emphasizing the low frequency end and leading to large resistive impedance values, but fortunately only in a frequency range of little or no relevance to beam stability. The presence of several layers renders the association of the impedance with a particular layer difficult. Nevertheless one notes that the real part of the coupling impedance is over a wide frequency range primarily determined by the dc resistance of the coating, but at very low frequencies it is lowered by the presence of the bypass. Again the impedance at very low frequencies is determined by the dc resistance of coating and bypass in parallel.
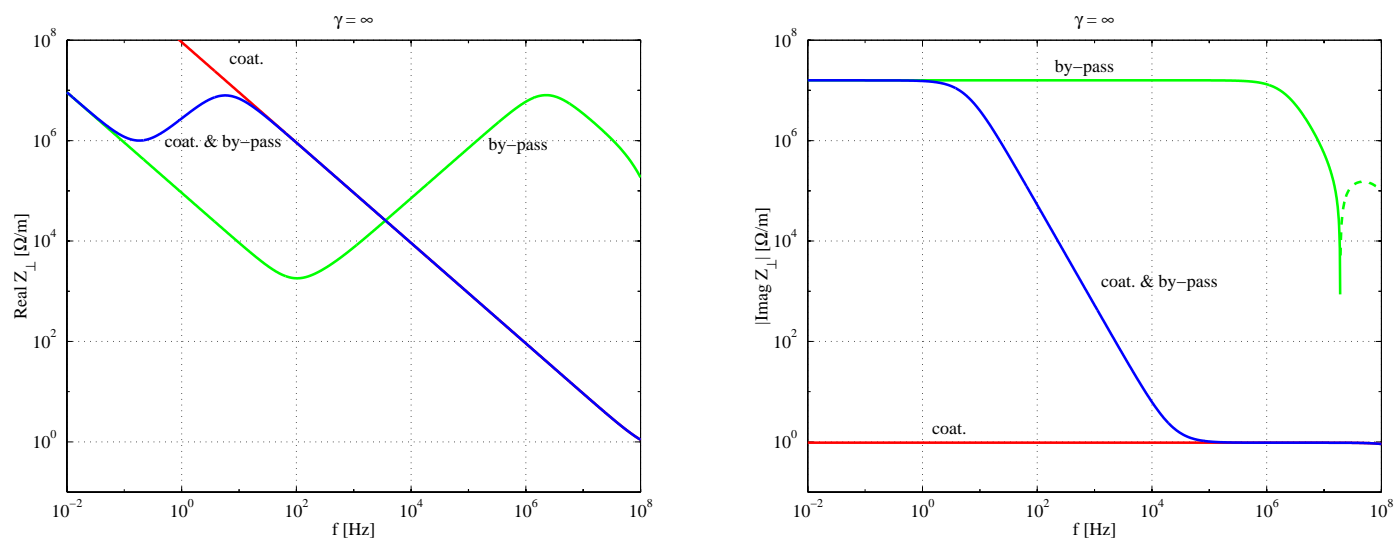

Fig.9. The transverse coupling impedance of a rectangular kicker model with various configurations of resistive coating and bypass.

\section{Acknowledgements}

It is a pleasure to acknowledge the stimulating e-mail correspondence with Dr. Caspers and the helpful discussions with Dr. Gluckstern. 


\section{References}

1. B. Zotter, Part. Accelerators, 1, p. 311 (1970)

2. A. Piwinski, Proc. 1977 PAC, Chicago, IL, p. 1364

3. B. W. Zotter and S. A. Kheifets, Impedances and Wakes in High-Energy Accelerators, (Word Scientific, Singapore, 1998)

4. A. W. Chao, Physics of Collective Beam Instabilities in High-Energy Accelerators, (J. Wiley \& Sons, New York, 1993)

5. A. Piwinski, in A.W. Chao and M. Tigner, eds. Handbook of Accelerator Physics and Engineering, (World Scientific, Singapore, 1999) p. 203

6. H. Hahn and A. Ratti, Report AD/RHIC/RD-105 (BNL, 1996)

7. F. Caspers, G. Dome, C. Gonzalez, E. Jensen, E. Keil, M. Morvillo, F. Ruggiero, G. Schröder, B. Zotter, and M. D'Yachkov, Proc. 1999 PAC, New York, p.1408

8. R. Gluckstern and B. Zotter, Phys.Rev. SPT-AB, 4,024402 (2001)

9. V. Danilov, S. Henderson, A. Burov, and V. Lebedev, SNS/AP Technical Note 14 (ORNL, 2001)

10. A. Piwinski, Reports DESY 84-097 (1984) \& DESY HERA 92-4

11. H.Henke and O. Napoly, Proc. $2^{\text {nd }}$ EPAC 90, Nice, p.1046

12. R.L. Gluckstern, Report CERN 2000-011 (2000)

13. B. Zotter and F. Sacherer, Report CERN 77-13 (1977) p.175

14. ref. 3 , p. 158

15. H. Hahn and S. Zatz, Proc. 1979 PAC, San Francisco, CA, p. 3626

16. H. Tsutsui, Report CERN-SL-2000-004 AP

17. W. von Koppenfels and F. Stallmann, Praxis der konformen Abbildung, (Springer-Verlag, Berlin, 1959) p.81

18. W.K.H. Panofsky and W.A. Wenzel, Rev. Sci. Instrum. 27, 967 (1956) 